\title{
Home Area Network (HAN) and Peripheral Devices using LV of Power Line Communication
}

\author{
Aaron Don M. Africa, Tun Tun Oo \\ Department of Electronics and Communications Engineering \\ De La Salle University, Manila \\ 2401 Taft Ave., Malate, Manila 1004, \\ Philippines, aaron.africa@dlsu.edu.ph
}

\begin{abstract}
This developed the Home Area Network (HAN) and integrate the network and devices into the house infrastructure. Combine with the infrastructure of the home network, the internal structure of peripheral devices, and the idea of LV power line communication. The using protocols are seven layers of OSI model or TCP/IP such as application, presentation, session, transport, network, datalink, and physical. This system is used for power line communication carried technology instead of wireless communication and CAT 6 wired communication by avoiding rewire troubleshooting. It is convenient for nodes to join and depart from the network. The experiment shows that the network has great reliability and is accessible to a general home network. HAN system, as same as LAN, was communicated with the Power Line Communication (PLC) Method. There are two types of HAN communication that it is wired and wireless. This paper using the wire communication PLC instead of the CAT 6 cable. However, the system was connected to the modem (modulation and demodulation) to the electric devices, which were operated by the computer.
\end{abstract}

Key words: Home Networks, Power Line Communications, OSI Model, TCP/IP

\section{INTRODUCTION}

Power Line Communication is a new technology for providing to access high-speed communication Internet or data communication. There are three main parts of the power line communication system (PLC) such as High Voltage, Medium Voltage, and Low Voltage. This paper uses the technology and devices for low voltage transmission at home power that it is possible to access the Internet in a very cost-effective way $[1,2,3]$. This is not the same ass the UTP, STP, or coaxial cable because the home power cable structure was optimized the frequency $(50 \mathrm{~Hz}$ or $60 \mathrm{~Hz})$, and Lowe Power lines present a varied environment for high-frequency communication signals.

Power Line Communication (PLC) is the technology that allows Internet or data transmission over the Electrical Power
Line network. Electricity Power Line can be used the home power (Lowe voltage) $22060 \mathrm{~Hz}$ or the national high voltage transmission grid. The data or Internet can be transferred is 200 Mbps speed via the house power line network. This speed can receive or transmit video transmissions such as Cable TV, CCTV, Voice such as PBX or VOIP (Voice Over Internet Protocol), data, and any other services that can be transmitted successfully. PLC use also in DC lines also has applications. There is two application to use PLC system over the DC transmission and distribution lines such as airplane that it is controlled electronic controls (UAV), train and automobiles [4,5]. This application can reduce wiring complexity, weight, and ultimately cost of communication inside vehicles. However, in this paper, the author will be dealing mostly with the Home Area network over AC LV lines.

Earlier, PLC is widely used in Smart Grid Technology and Smart tie Inverters. As the markets, PLC is seen to other application forms such as traffic light control, UPS by communicating to a network device, vending machines, reception to room management of the hotel, telemetry, and electronic transportation.

Smart home appliance and power line network and control system to make the home ready for smart grid integration. Indeed, more and more insight and new contribution ideas for the world of Power Line Communication [6,7].

\section{MODULATION AND DEMODULATION OF PLC SYSTEM}

In this paper, PLC communication is used the digital and Analog modulation or combining that it is the main factor in PLC communication. Almost modulation is digital modulation in the PLC modem. Digital modulation is dependent on digital signal processing such as at the receiver to transformation (DAC) digital to analog converters and at the transmitter analog to digital converters (ADC) to transform analog information into a digital. Figure 1 shows the Modulation and Demodulation of Digital communication system. 


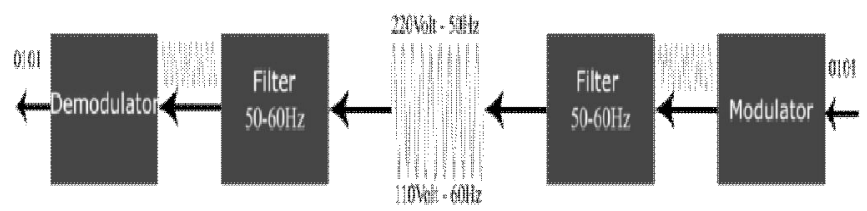

Figure 1: The Block Diagram of Modulation and Demodulation of Digital communication system

From right to left, the digital data is transmitted by combining modulation that digital bits are converted to analog frequency/sine wave. E.g. Bit 1 is converted $10 \mathrm{kHz}$ or bit 0 is sine wave $20 \mathrm{kHz}$. But the main frequency signal is used $50 / 60 \mathrm{~Hz}$ signal that it is filtered at the filter. The transmitted data are free to travel via the main power line. When they find their destination to another 50 to $60 \mathrm{~Hz}$ filter is involved. After the filter pass, the remaining signal is the modulated transmitted data. These signals are demodulated and converted that it is also converted return digital bits [8].

There are many types of digital modulation system. Some of the modulation schemes are (OFDM) Orthogonal Frequency Division Multiplexing, (BPSK) Binary Phase Shift Keying (BPSK), (FSK) Frequency Shift Keying (FSK), (S-FSK) Spread-FSK [9]. Table 1 shows the modulation comparisons.

Table 1: Comparison modulations of two important criteria

\begin{tabular}{|c|c|c|}
\hline $\begin{array}{c}\text { Modulation } \\
\text { Scheme }\end{array}$ & $\begin{array}{c}\text { Bandwidth } \\
\text { efficiency }\end{array}$ & Complexity \\
\hline BPSK & Medium & Low \\
\hline FSK & Medium & Low \\
\hline SFSK & Low & Medium \\
\hline OFDM & High & High \\
\hline
\end{tabular}

This paper presents a smart home network that integrates an OFDM offers high data rates. However, Fast Fourier Transforms (FFT) and Inverse-FFT (IFFT) are operated that it is required computational horsepower. Figures 2,3,4,5,6 and 7 shows the modulation diagrams and system outputs of ASK, QPSK, OFDM, and QPSK BER /SER simulations.

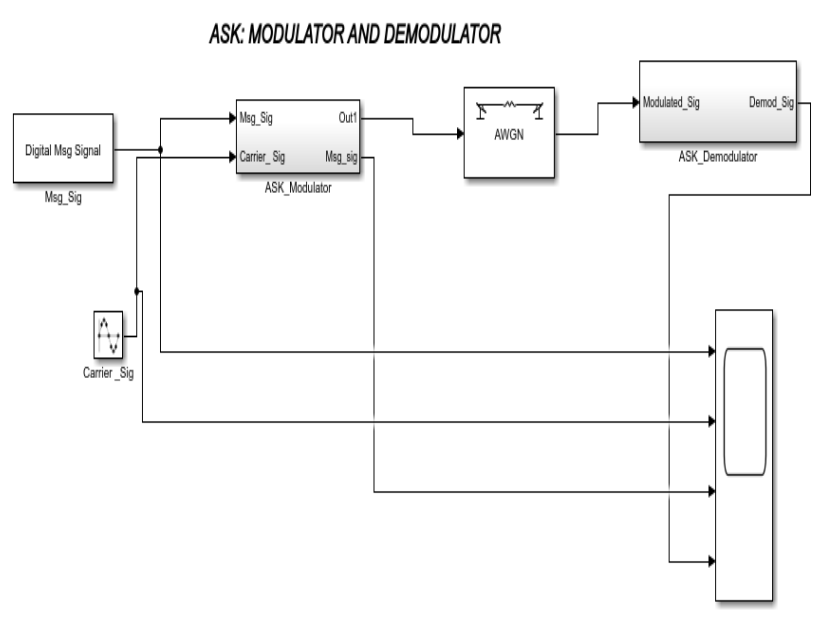

Figure 2:ASK modulation/demodulation Simulation Matlab

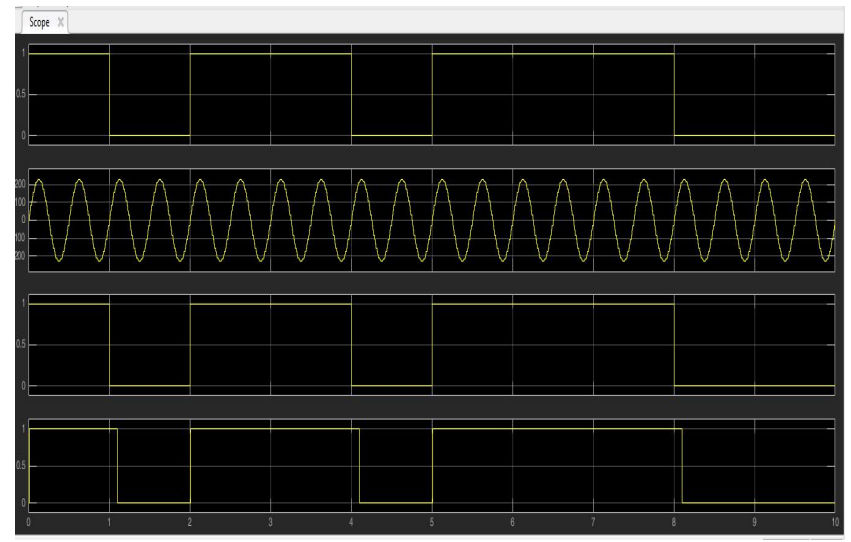

Figure 3: ASK Output/Input Waveform Simulation Matlab

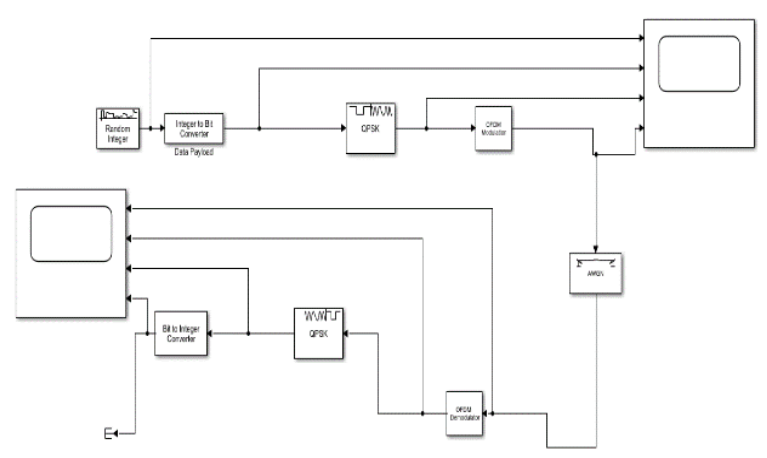

Figure 4: QPSK \& OFDM Modulation/Demodulation simulation

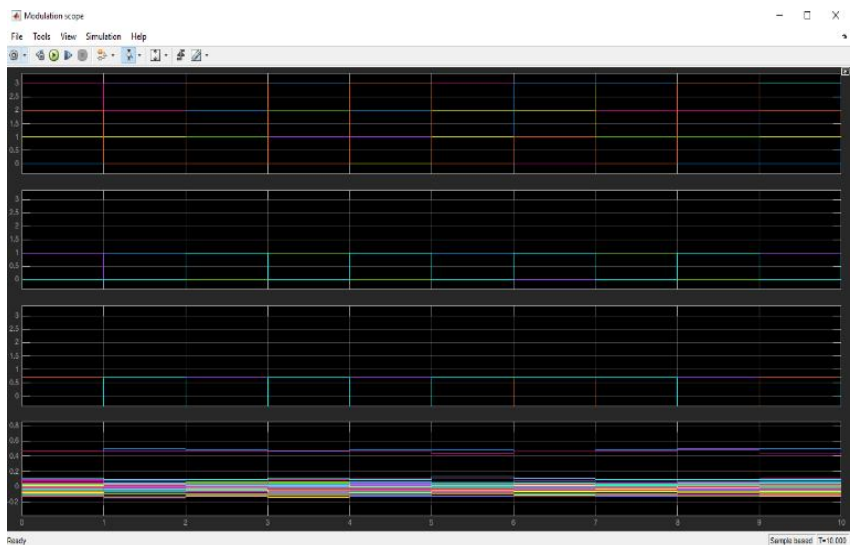

Figure 5: QPSK \& OFDM Modulation Output Waveform

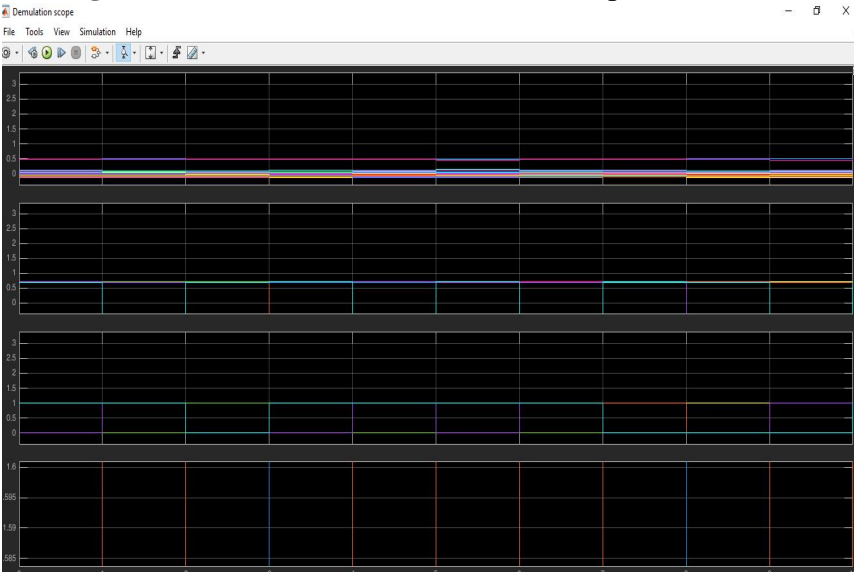

Figure 6: QPSK \& OFDM Demodulation Output Waveform 


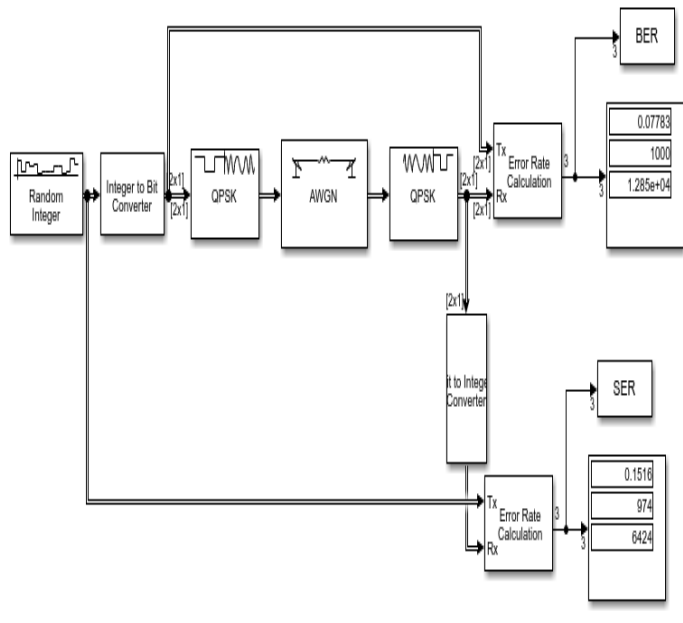

Figure 7: QPSK BER /SER Noise simulation

\section{PLC ADAPTOR OR MODEM}

The electrical wiring of the home is not the 21 st century by sending signals via an electrical cable. The electrical power distribution companies have been transmitted control data signals over the main since 1920. A variety of electrical frequencies can be supported at the electrical wiring that it is used $50 / 60 \mathrm{~Hz}$ signals, can transit extra data via the duplicate electrical wiring at higher frequencies without inducing each obstruction [12,13]. The IEEE assigned number IEEE 1901.

Figure 8 shows the Sample Configuration of Power Line Adapter.

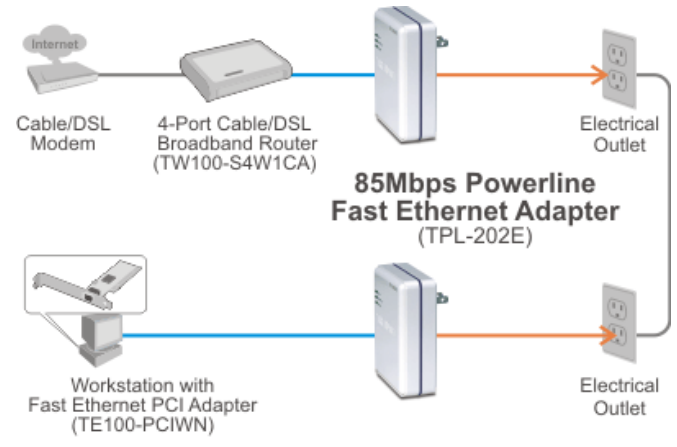

Figure 8:Sample Configuration of Power Line Adapter

The power line network is less visible than it is called no new wires. The Ethernet cables are connected to the router and the power line adapters. And then, these adapters plus the nearest power socket. The second adapter plus into the nearest power socket that it would be used the Internet or data communication [10,11]. The adapters are auto-detecting to each other and auto-connect, enabling data packets to the router. The data packet is transporting into the first adapter via the Ethernet cable across the electrical wiring system in the home. And then, this data and the electrical signal are outed into the second adapter across the electrical wiring at this home and separated data and electrical signal. Figure 9 shows the Configuration of Power Line Adapter.

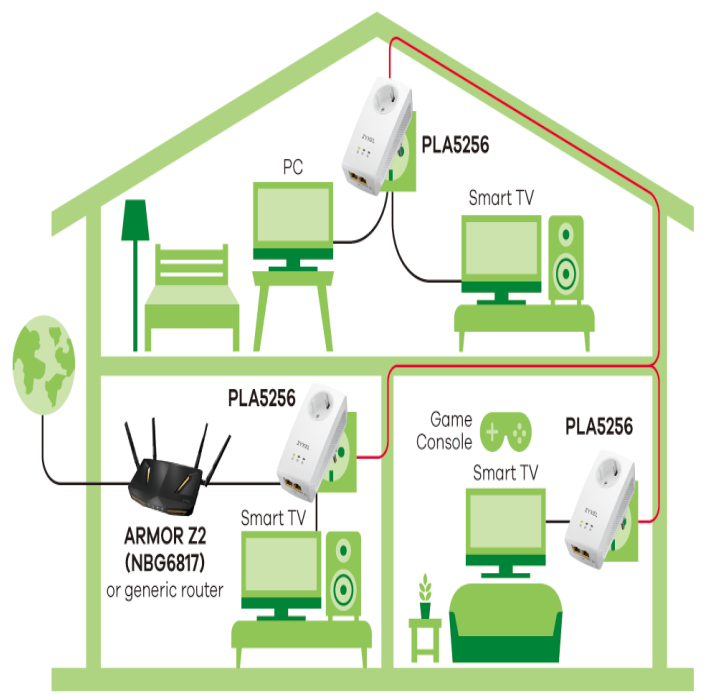

Figure 9:Configuration of Power Line Adapter

Figures 10 and 11 shows the PLC Modem Internal Block Diagram and Power Line Communication Systems.

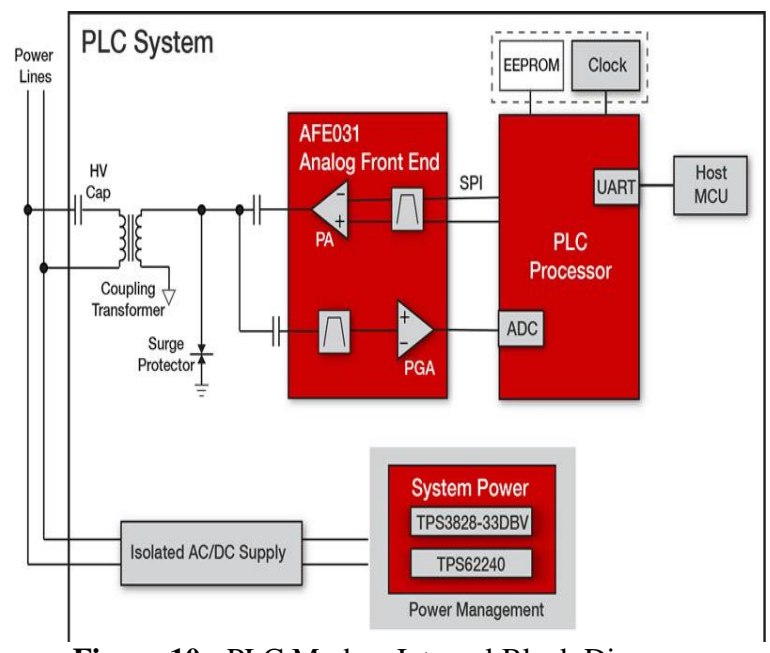

Figure 10: PLC Modem Internal Block Diagram

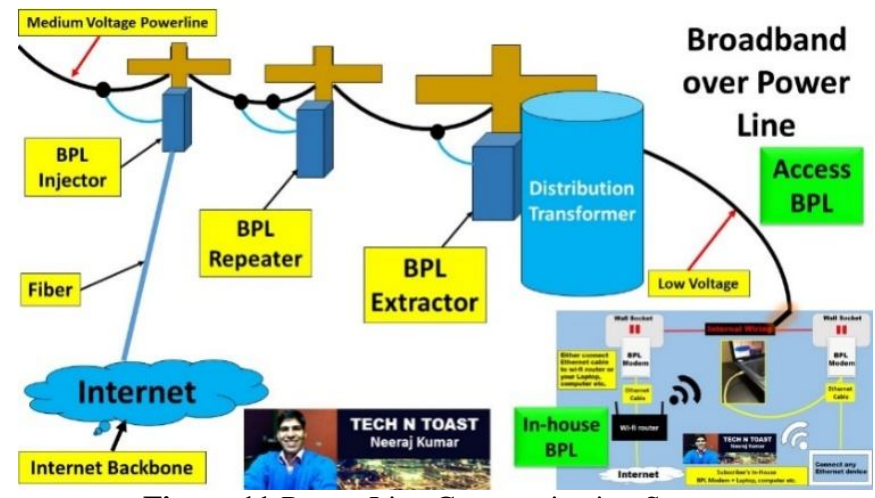

Figure 11:Power Line Communication Systems 


\section{HAN AND SMART HOME AUTOMATION}

Power Line Communication is the physical layer of the OSI model that it is used transmission media such as wire such as fiber or microwave. Therefore, attenuation, losses, and SNR are given trouble to the systems of PLC. And then, EMI, capacitance, impedance matching, and loading balance are also considered because these noises are affected by any communication system. That why Power Line Communication technologies are delay improving. If the Power Line Communication (PLC) technology is progressed, everywhere is used equipment and peripheral devices, PLC will create unlimited to improve over the communication. Figure 12 shows the Home Area Network using PLC.

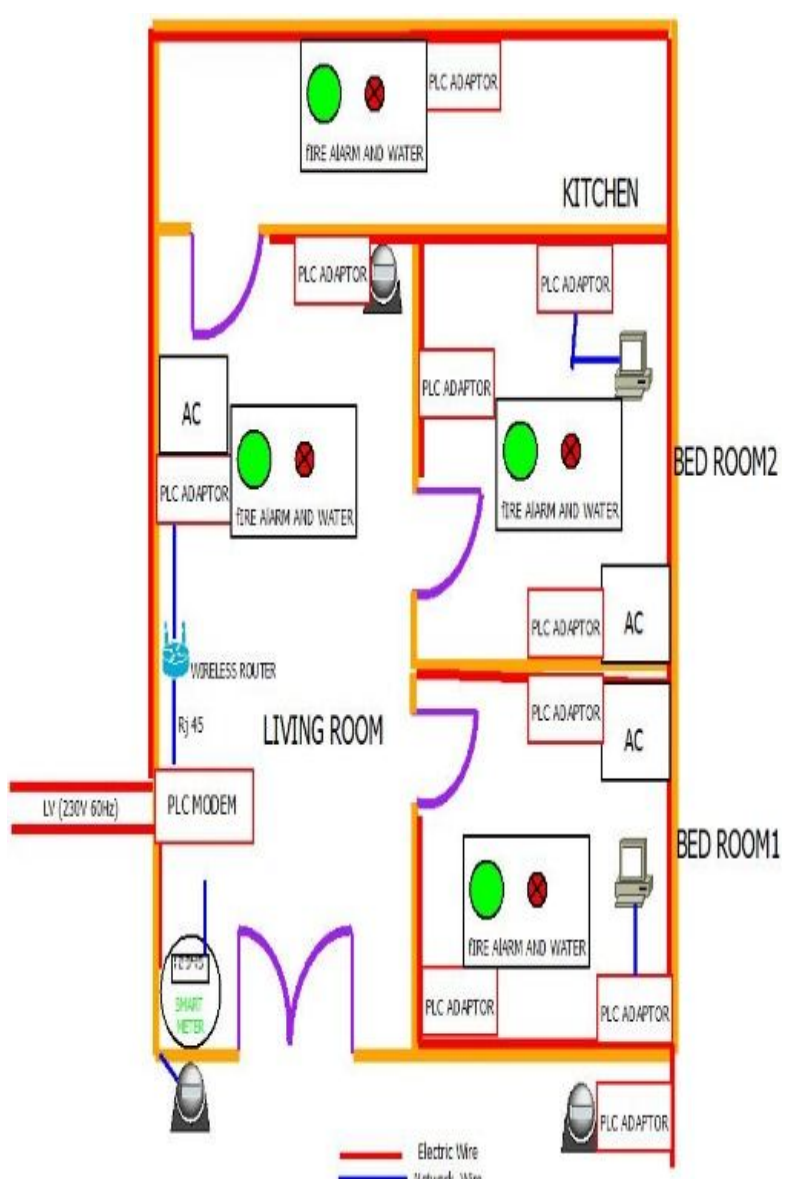

Figure 12: Home Area Network using PLC

The Lower Voltage power line has transmitted with the Data and Electricity such as AC $230 \mathrm{~V} 60 \mathrm{~Hz}$ and $2 \mathrm{Mbps}$ data rate. Firstly, PLC MODEM has accepted the electricity with data, and then it will separate Data and Electricity. After that, Data Line that is the output of LAN cable from PLC MODEM to the Cisco Wireless Router. Cisco Wireless Router would be used LAN and WLAN with efficient and powerful data speed. And then, PLC Adapter was transmitted data to use the all electricity socket that it can use the data and electricity. The fire alarm and water sprinkler were used for speaking detection and fire detection by using PLC adapter because it is more useful not more need to add LAN cable and do not consider LAN cable's limitation length. All AC (Air
Condition) is controlled by using PLC networking and IoT (Internet of Things). So, the phone software can control or off and wind speed sensors can be controlled cool or hot conditions. Figure 13 shows the Smart Home Automation.

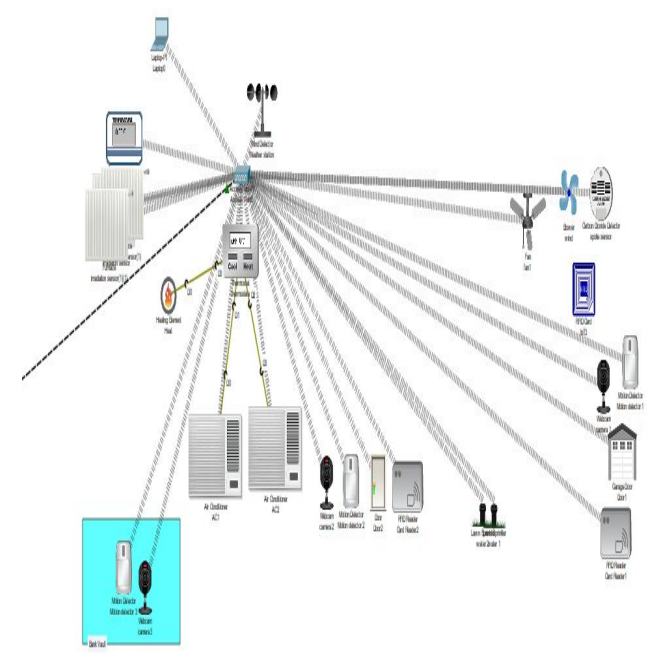

Figure 13: Smart Home Automation

There are five separates operations in smart home automation. Firstly, Gate door that it is the main gate of the home is controlled by the RFID card and motion sensor. The motion sensor that is connected to CCTV has detected motion activities. If the moving objects are be detected from the motion sensor, CCTV camera would be taken a photo of moving objects which it is captured the video another time. RFID (Radio Frequency ID) card is shown the match ID or not and then this card is identified from the storage ID system. If the corrected valid ID card detected, the gate would be open. If no valid ID card detected, the gate is never open the gate. The second operation is the door of a home that is the same operation as the main gate system. The third operation is the money vault room. This room is prevented from thieves and other unnecessary issues. This room is installed on the motion sensor and camera. Besides, moving everybody or every object in this room would take the photo by the system. Forth operation is the kitchen room. This room is sensed the $\mathrm{CO} 2$ from the cooking and another smoke. When either over the normal cook smoking or below firing smoking was detected, the fan and air blower fan are operated to release internal smoke to the outside. The fifth operation is the Aircon, Heat, and Water sprinkler of grasses field. The temperature sensor is sensing the outside weather heat. If the temperature is above $23^{\circ} \mathrm{C}$, the water sprinkler of grasses field will be a spray of water, and all $\mathrm{AC}$ open. If the temperature is below $20^{\circ} \mathrm{C}$, all Aircon is close and the water sprinkler is stopped. Figure 14 shows the Cisco routing of Home Area Network. 


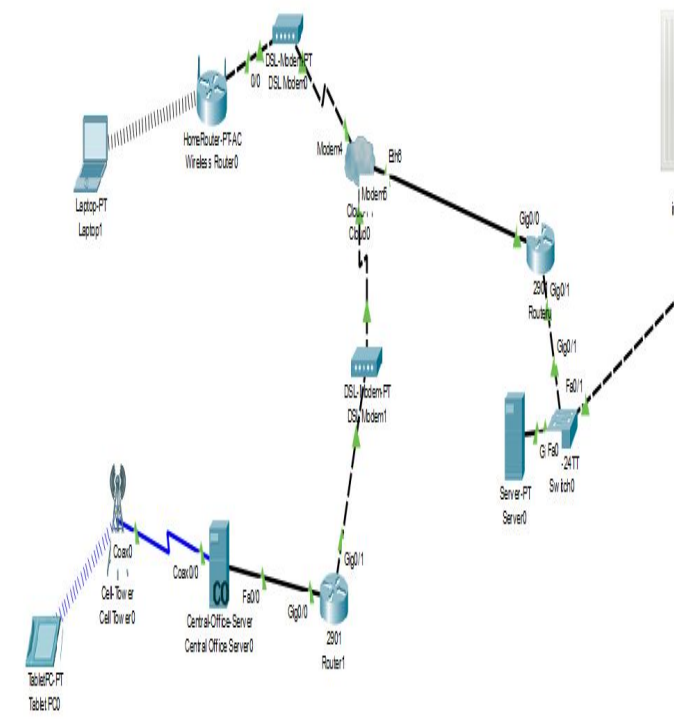

Figure 14: Cisco routing of Home Area Network

The Cisco router is used with a remote server and is connected to the cloud. Besides, the office and mobile phone can be the interface to the home network or home automation service via the cloud. Figure 15 shows the Fire Alarm system of Home Area Network.

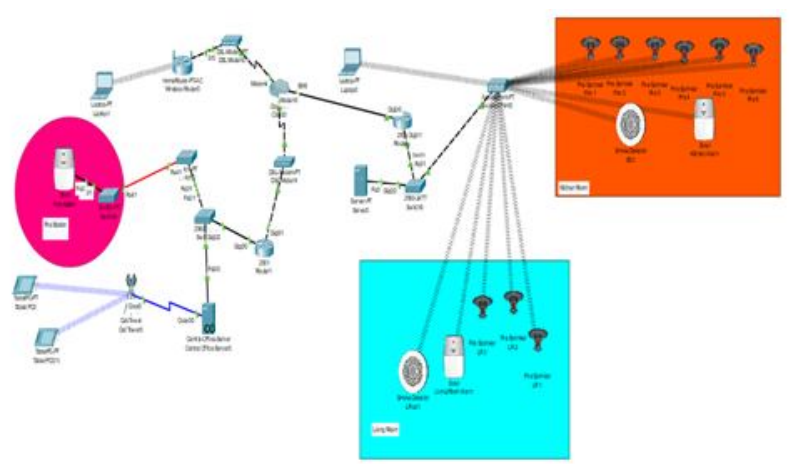

Figure 15: The Fire Alarm system of Home Area Network

When the Smoking detector in the kitchen is detected $\mathrm{CO} 2$ from extremely above of the cooking smoke, the fire alarm and water sprinkler is operated and then fire alarm at the fire station is alert that the fireman will know where the fire is burned. Moreover, the Living room is the same process as the kitchen.

\section{HAN DISCUSSIONS}

If the PLC technologies are more widely improved, the useful applicants will be improved and helpful technology. The applications are progressed unlimitedly and completely. The IoT (Internet of Things) network is very advanced and solved many problems out easily that it is saving a lot of time. This application can easily connect with the other one or with any other devices. The smartphones and other devices like computers, tablets can control and manage the systems securely that it is used the IoT network. For optimization Fuzzy Logic can be used $[14,15,16]$.

All devices that it is used PLC technology is normally worked physical layers. If all of the devices are worked two layers and three layers, they will be saving the attenuation, data loss, and other noise. Layer two (Datalink) is operated MAC (Media Access Control) and LLC (Logical Link Control) with Frame and layer three (network) is operated IP (Internet Protocols) with packets.

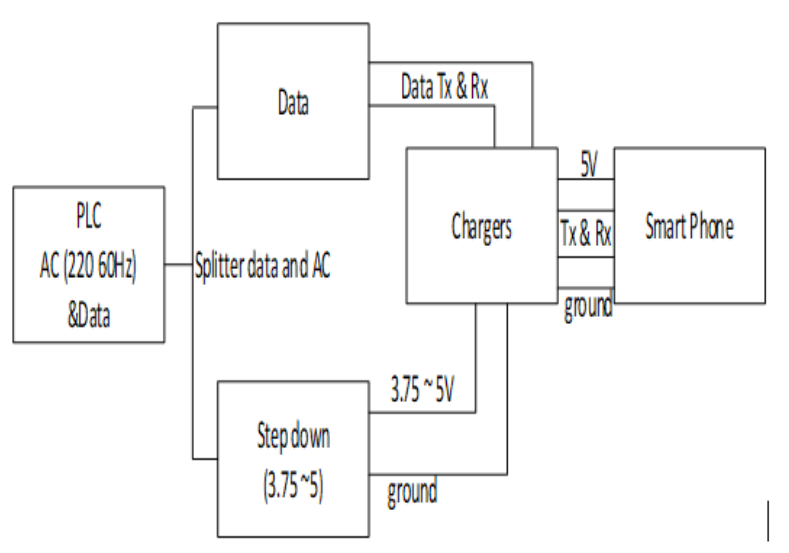

Figure 16: Data using while charging to the phone

If the PLC technology transmission system is improved, the smartphone will change the charging adaptor. The adapter will be used by combining data and charging. When the smartphone will be charging, the data or Internet is used because the charging USB cable has four lines wire. Moreover, two lines used the positive voltage and ground, and then another two lines are data transmitted and received.

\section{CONCLUSION}

The power line communication that it has many reasons to use gets strong and compact communication with the highest speed data. Other essential is attenuation largely because the LF (electricity frequency) range and HF with the highest speed data range combine over the transmission line. However, the user will get many benefits by using Power line communication. The smart home with PLC operates over the one wire that it is combine electrical signal and data connection. Therefore, this system did not need to add any network cable or powerline cord for connecting all devices easily successfully. The author's design using a PLC system will reduce the cost effectively or other necessary wire costs and compatible to use.

\section{REFERENCES}

[1] F. A.Pinto-Benel and F. Cruz-Roldán, "A bandpass wavelet OFDM system for power line communications," Journal of the Franklin Institute. 2020. 
[2] K. Sharma and L. M. Saini, "Power-line communications for smart grid: Progress, challenges, opportunities and status," Renewable and Sustainable Energy Reviews. Vol. 67,pp. 704-751, 2017. https://doi.org/10.1016/j.rser.2016.09.019

[3] E. Meza and J. Aspiazu, "Design of a Power Line Communications Transceiver Based on OFDM," Procedia Technology. Vol. 17, pp. 107-113, 2014.

[4] S. Mudriievskyi, "Power Line Communications: State of the art in research, development and application," AEU International Journal of Electronics and Communications. Vol. 68, No. 7, pp. 575-577, 2014. https://doi.org/10.1016/j.aeue.2014.04.003

[5] M. Mizutani, Y. Miyoshi, K. Tsukamoto, M. Tsuru and Y. Oie,"Network-supported TCP rate control for high-speed power line communications environments," Simulation Modelling Practice and Theory. Vol. 19, No. 1, pp. 69-83, 2011. https://doi.org/10.1016/j.simpat.2010.06.017

[6] T. Oo and A.Africa,"Design and implementation of bandwidth monitoring, line aggregation of VoIP," International Journal of Advanced Trends in Computer Science and Engineering. Vol. 8, No. 4, pp. 1326-1331,2019. https://doi.org/10.30534/ijatcse/2019/46842019

[7] H. Singh, and S.Neware, "Improving customer segmentation in e-commerce using predictive neural network," International Journal of Advanced Trends in Computer Science and Engineering. Vol. 9, No. 2, pp. 2326-2331, 2020.

https://doi.org/10.30534/ijatcse/2020/215922020

[8] K. Raman, R. Hablani and S. Randive, "Image analysis of PLC diagnosis system," International Journal of Advanced Trends in Computer Science and Engineering.Vol. 9, No. 2, pp. 1973-1977,2020. https://doi.org/10.30534/ijatcse/2020/165922020

[9] M. Khalaf, A. Wahab, S. Alhady, H. Husin and W. Othman, "Recognition of digitally modulated signal by ann algorithm," International Journal of Advanced Trends in Computer Science and Engineering. Vol. 9, No. 2,pp. 2414-2421, 2020. https://doi.org/10.30534/ijatcse/2020/227922020

[10] R. Sara, and S.Neware, "Orange fruit disease classification using deep learning approach," International Journal of Advanced Trends in Computer Science and Engineering. Vol. 9, No. 2, pp. 2297-2301, 2020. https://doi.org/10.30534/ijatcse/2020/211922020

[11] H. Li, D. Liu, J. Li and P. Stoica, "Channel order and RMS delay spread estimation with application to AC power line communications,"Digital Signal Processing. Vol. 13, No. 2,pp. 284-300, 2003.

[12] X. Gao, K. Yang, N. Yang and J. Wu,"Energy-efficient resource block assignment and power control for underlay device-to-device communications in multi-cell networks," Computer Networks. Vol. 149, pp. 240-251, 2019.

[13] Y. Bian, Y. Li, D. Zheng, W. Li, X. Hong, J. Qiu and J. $\mathrm{Wu}$,"Low-complexity parallel real-valued weight adaptive digital combining algorithm for coherent FSO communications employing modes diversity reception under atmospheric turbulence channel," Optics Communications. Vol. 474,2020.

https://doi.org/10.1016/j.optcom.2020.126078

[14] C. Escolar-Jimenez, K. Matsuzaki and R.Gustilo, "A neural-fuzzy network approach to employee performance evaluation," International Journal of Advanced Trends in Computer Science and Engineering. Vol. 8, No. 3, pp. 573-581, 2019. https://doi.org/10.30534/ijatcse/2019/37832019

[15]C. Escolar-Jimenez, K. Matsuzaki and R.Gustilo, "Intelligent shortlisting process for job applicants using fuzzy logic-based profiling," International Journal of Advanced Trends in Computer Science and Engineering. Vol. 8, No. 3, pp. 567-572, 2019. https://doi.org/10.30534/ijatcse/2019/36832019

[16]C. Escolar-Jimenez, K. Matsuzaki, K. Okada and R.Gustilo, "Data-driven decisions in employee compensation utilizing a neuro-fuzzy inference system," International Journal of Emerging Trends in Engineering Research. Vol. 7, No. 8, pp. 163-169, 2019. https://doi.org/10.30534/ijeter/2019/10782019 